EPSC Abstracts

Vol. 15, EPSC2021-561, 2021

https://doi.org/10.5194/epsc2021-561

Europlanet Science Congress 2021

(C) Author(s) 2021. This work is distributed under

the Creative Commons Attribution 4.0 License.

\title{
A New Facility for the Planetary Science Community at DLR: the Planetary Sample Analysis Laboratory (SAL).
}

Enrica Bonato, Sabrina Schwinger, Alessandro Maturilli, and Jörn Helbert

Deutsches Zentrum für Luft- und Raumfahrt (DLR), Planetary Laboratories Department, Berlin, Germany (enrica.bonato@dlr.de)

Introduction: Laboratory measurements of extra-terrestrial materials like meteorites and ultimately materials from sample return missions can significantly enhance the scientific return of the global remote sensing data.

This motivated the addition of a dedicated Sample Analysis Laboratory (SAL) to complement the work of well established facilities like the Planetary Spectroscopy Laboratory (PSL) and the Astrobiology Laboratories within the Department of Planetary Laboratories at DLR, Berlin. SAL is being developed in preparation to receive samples from sample return missions such as JAXA Hayabusa 2 and MMX missions, the Chinese Chang-E 5 and 6 missions as well as the NASA OsirisREX mission. SAL will be focusing on spectroscopic, geochemical, mineralogical analyses at microscopic level with the ultimate aim to derive information on the formation and evolution of planetary bodies and surfaces, search for traces of organic materials or even traces of extinct or extant life and presence of water.

Sample Analysis Laboratory: The near-term goalis to set up the facilities on time to receive samples from the Hayabusa 2 mission. The operations have already started in 2018 with the acquisition of a vis-IR-microscope and it will continue with the acquisition of: Field Emission Gun scanning electron microscope (FEG-SEM), Field Emission Gun - electron microprobe analyser (FEGEMPA), X-ray diffraction (XRD) system with interchangeable optics for $\mu$ XRD analysis anda polarised light microscope for high resolution imaging and mapping

The facilities will be hosted in a clean room (ISO 5) equipped with glove boxes and micromanipulators to handle and prepare samples. All samples will be stored under dry nitrogen and can be transported between the instruments with dedicated shuttles in order to avoid them to enter in contact with the external environment. Based on current planning the first parts of SAL will be operational and ready for certification by end of 2022 .

Current facilities: To characterize and analyse the returned samples, SAL facilities will work jointly with the existing spectroscopic capabilities of PLL.

PLL has the only spectroscopic infrastructure in the world with the capability to measure emissivity of powder materials, in air or in vacuum, from low to very high temperatures [1-3], over an extended spectral range from 0.2 to $200 \mu \mathrm{m}$. Emissivity measurements are complemented by reflectance and transmittance measurements produced simultaneously with the same set-up. Recently a vis-IR-microscope was added to extend spectral analysis to the sub-micron scale. In addition, the department is operating a Raman micro-spectrometer with a spot size on the sample in 
focus of $<1.5 \mu \mathrm{m}$. The spectrometer is equipped with a cryostat serving as a planetary simulation chamber which permits simulation of environmental conditions on icy moons and planetary surfaces.

PLL leads MERTIS on BepiColombo as well as the BioSign exposure experiment on the ISS. The labs have performed laboratory measurements for nearly every planetary remote sensing mission. PLL has team members on instruments on the MarsExpress, VenusExpress, MESSENGER and JAXA Hayabusa 2 and MMX missions. Most recently we joined the Hayabusa 2 Initial Sample Analysis Team.The samples analyzed at PLL range from rocks, minerals, meteorites and Apollo and Luna lunar soil samples to biological samples (e.g. pigments, cell wall molecules, lichens, bacteria, archaea and other) and samples returned from the ISS (BIOMEX) $[4,5,6]$ and the asteroid Itokawa (Hayabusa sample).

PLL is part of the "Distribute Planetary Simulation Facility" in European Union funded EuroPlanet Research Infrastructure (http://www.europlanet-2020-ri.eu/). Through this program (and its predecessor) over the last 9 years more than 80 external scientists have obtained time to use the PLL facilities. PLL has setup all necessary protocols to support visiting scientist, help with sample preparation, and archive the obtained data.

Outlook: DLR has started establishing a Sample Analysis Laboratory. Following the approach of a distributed European sample analysis and curation facility as discussed in the preliminary recommendations of EuroCares (http://www.euro-cares.eu/) the facility at DLR could be expanded to a curation facility. The timeline for this extension will be based on the planning of sample return missions. The details will depend on the nature of the returned samples. Moreover, SAL will be running in close cooperation with the Museum für Naturkunde in Berlin and it will be operated as a community facility (e.g. Europlanet), supporting the larger German and European sample analysis community.

References: [1] Ferrari et al., Am. Min., (2014), 99(4): p. 786-792. [2] Maturilli and Helbert, JARS (2014), 8(1): p. 084985. [3] A. Maturilli, et al., (2019) Infrared Remote Sensing and Instrumentation XXVII, 10.1117/12.2529266. [4] de Vera et al. (2012), PSS, 74(1): p. 103-110. [5] Serrano et al. (2014), PSS, 98: 191-197. [6] Serrano et al. (2015), FEMS Microbiology Ecology, 91(12): 2015, fiv126. 